\title{
Transfer Öğrenme ve Çekişmeli Üretici Ă̆ Yaklaşımlarını Kullanarak Cilt Lezyonu Sınıflandırma Doğruluğunun İyileştirilmesi
}

\author{
Kazım FIRILDAK ${ }^{*}$, Gaffari ÇELIK ${ }^{2}$, M. Fatih TALU ${ }^{3}$ \\ ${ }^{1}$ Bilgisayar Teknolojileri, Kovancılar Meslek Yüksek Okulu, Fırat Üniversitesi, Elazığ, Türkiye \\ ${ }^{2}$ Bilgisayar Teknolojileri, Meslek Yüksek Okulu, Ağrı İbrahim Çeçen Üniversitesi, Ağrı, Türkiye \\ ${ }^{3}$ Bilgisayar Mühendisliği, Mühendislik Fakültesi, İnönü Üniversite, Malatya, Türkiye \\ ${ }^{{ }^{* 1}}$ kfirildak@ firat.edu.tr, ${ }^{2}$ gcelik@ agri.edu.tr, ${ }^{3}$ fatihtalu@ @mail.com
}

(Geliş/Received: 06/10/2020;

Kabul/Accepted: 05/04/2020)

\begin{abstract}
Öz: Bu çalışmada, en yaygın kanser türlerinden biri olan cilt kanseri imgelerinin sınıflandırılmasına odaklanılmıştır. Yapılan araştırma sonucunda cilt kanseriyle ilgili literatürdeki en kapsamlı etiketlenmiş veri kümesinin HAM10000 olduğu görülmüştür. 7 farklı lezyon türüne ait 10.000 'den fazla etiketli imge içeren bu veri kümesinin klasik Evrişimsel Sinir Ağlarıyla (ESA) sınıflandırma doğruluğunun arttırılması amaçlanmaktadır. Bu makalede, mevcut iki farklı tekniğin (transfer öğrenme ve imge üretimi) lezyon sınıflandırma doğruluğuna etkisi incelenmiştir. Birinci teknik, cilt lezyonu veri kümesini sınıflandırmak için tasarlanan yeni bir ESA'ya, ImageNet veri kümesiyle eğitilmiş AlexNET ağındaki parametrelerin kısmi ve tam transfer yoluyla aktarılmasıdır. İkinci teknik, gerçek lezyon imgelerinden imge üretilmesiyle veri kümesinin genişletilmesidir. $\mathrm{Bu}$ genişletme işleminde klasik üretme ve Çekişmeli Üretici Ağ (ÇÜA) tekniklerinin başarımları değerlendirilmiștir. Yapılan deneysel çalıșmalar neticesinde, kısmi parametre transferi ve Derin Evrişimsel Çekişmeli Üretici A $\breve{g}$ (DEÇÜA) temelli imge üretim tekniği kullanılarak veri kümesinin genişletilmesi yaklaşımlarının birlikte kullanılması en yüksek lezyon sınıflandırma doğruluğunu (\%93) vermiştir. Yöntemler, literatürdeki güncel yöntemle kıyaslanarak toplam doğruluk başarımındaki üstünlüğü gösterilmiştir.
\end{abstract}

Anahtar kelimeler: Çekişmeli Üretici Ağlar, Cilt Kanserinin Tanı ve Tespiti, Çekişmeli Üretici Ağlar ile Veri Üretimi, Evrişimsel Sinir Ağları

\section{Improving Skin Lesion Classification Accuracy Using Transfer Learning And Generative Adversarial Network}

\begin{abstract}
This study focuses on the classification of skin cancer images, one of the most common types of cancer. As a result of the research, it was found that the most comprehensive labeled data set in the literature related to skin cancer were HAM10000. This data set, which contains more than 10,000 tagged images of 7 different lesion types, is aim to increase accuracy in classification with Convolutional Neural Networks (CNNs). In this study, the effect of two different techniques (transfer learning and image production) on lesion classification accuracy was investigated. The first technique is the partial and full transfer of parameters in the ImageNet image set-trained AlexNET network to a new CNN designed to classify the skin lesion data set. The second technique is to expand the dataset by generating images from true lesion images. In this expansion process, the performances of classical production and Generative Adversarial Network (GAN) techniques were evaluated. As a result of the experimental studies, the use of partial parameter transfer and data set expansion approaches using Deep Convolutional Generative Adversarial Network (DCGAN) based image generation technique yielded the highest lesion classification accuracy (93\%). The methods have been compared to the current method in the literature and total accuracy performance is demonstrated.
\end{abstract}

Key words: Generative Adversarial Network, Detection of Skin Cancer, Deep Convolutional Generative Adversarial Network Based Data Augmentation, Convolutional Neural Networks.

\section{Giriş}

Tıp alanındaki yapay öğrenme sistemlerinin temel problemleri arasında, yeterli veri sayısına sahip veri kümelerinin bulunmaması ve veri toplanmasında yaşanan zorluklar olarak özetlenmektedir [1]. Bunun yanında tıbbi verilerin etiketlenmesi ve sınıflanmasında uzman bilgisine ihtiyaç duyulmaktadır [1]. İhtiyaçları karşılayacak bir veri kümesi oluşturmak insan gücü, zaman, uzman bilgisi ve verinin ön işlenmesi gibi aşamaları ile tıp alanındaki yapay öğrenme araştırmacılarının çözmeleri gereken bir problemdir. Literatürde tıp alanında çalışılan bilgisayar bilimciler için sunulan veri kümeleri bulunuyor olsa da yapay öğrenme algoritmaları bu veri

\footnotetext{
* Sorumlu yazar: kfirildak@firat.edu.tr. Yazarların ORCID Numarası: ${ }^{1} 0000-0002-1958-3627,{ }^{2} 0000-0001-5658-9529,{ }^{3}$ 0000-0003-11668404
} 
kümeleri için her zaman iyi sonuçlar vermemektedir. Tüm bu bilgiler ışı̆̆ında etkili bir yapay öğrenme sistemine uygun bir veri kümesi oluşturmak için veri artırım yöntemleri önemli bir araştırma konusu haline gelmektedir.

Yapay öğrenme algoritmalarında eğitim ve test doğruluğu arttırmak için kullanılan iki temel veri artırım yöntemi bulunmaktadır. Bunların ilki klasik veri artırım yöntemleridir. Bu yöntemler Çevirme (Flipping), Döndürme (Rotation), Kırpma (Cropping), Kaydırma (Shifting) gibi verilerin geometrik özelliklerini ve renk özelliklerini değiştirerek yapay öğrenme yöntemlerinin doğruluk başarımını artırmaya çalışmaktadır [2]. Ayrıca veri üreten bir modelle, sentetik veri oluşturulması işlemi de klasik veri artırım yöntemleri arasında gösterilmektedir. Bu klasik veri artırım yöntemleri, yapay öğrenme uygulamalarında önemli başarılar sağlamaktadır [2]. İkinci bir veri artırımı yöntemi ise yapay öğrenme mimarilerinden olan ve oyun teorisinden ilham alınarak oluşturulan Çekişmeli Üretici Ağlardır (ÇÜA) [3]. Bu ağ modeli gerçek imgeler ile en benzer yeni gerçek imgeler oluşturması için eğitilen bir yapay öğrenme modelidir. Eğitim verisi kullanarak üretim yapan ÇÜA literatürde farklı modelleri önerilmiştir. Bunlar arasından stil transferi yapan [4], semantik boyama işlemi yapan [5] ve veri üretimi yaparak çeşitli problemlere çözüm ürettiği gösterilmiştir [1], [6].

Modern bir eğiticili öğrenme yaklaşımı olan ESA, medikal uygulamaların birçoğunda kullanılmıştır [1], [6]-[8]. Literatürde karaciğer lezyonlarının sınıflandırmasında ESA başarımını artırmak için ÇÜA kullanılmıştır. Böylelikle karaciğer lezyonların sınıflanmasında klasik veri artırım yöntemlerine göre duyarlılık (sensitivity) ve özgüllük (specificity) oranları $\% 3$ ila $\% 5$ arasında geliştirmişlerdir [1]. Xue ve diğ. Beyin tümörünün MRI imgeleri üzerinde bölütleme yapmak için ÇÜA faydalanmışlardır [9].

Dünyada yaygın olarak görülen kanser türlerinden olan cilt kanseri ABD'de yılda yaklaşık 10000 insanın ölümüne neden olmaktadır [10]. Lezyonların görsel olarak muayene edilerek tespit edilmesi bir çok literatür çalışmasında detaylı olarak gösterilmiştir [11], [12]. Bunun yanında bilgisayarlı görü ve yapay öğrenme algoritmalarıyla cilt lezyonlarının tespiti ve sınıflanmasında yaygın olarak kullanılmaktadır [12]-[14]. Cilt lezyon imgelerinin bölütlenmesi ve sınıflandırılmasında, ÇÜA kullanılarak veri artırımı yapılmış ve ESA'nın başarımı arttırılmıştır [12]. Ayrıca cilt lezyonlarındaki melanomu tanıyan ESA ile uzman tahminleri karşılaştırılmış ve öğrenme yönteminin tıp alanındaki başarımı detaylı olarak gösterilmiştir [13]. [14] nolu çalışmada, Res50 ve Res101 mimarileri kullanılarak cilt lezyonu imgelerinin öznitelikleri çıkarılmış ve Destek Vektör Makinesi (DVM) yöntemiyle \%89.9 doğrulukta sınıflandırılmıştır.

Bu makale çalışmasında, ilk olarak kullanılan veri kümesi (HAM10000 [15]) hakkında detaylı bilgi verilmektedir. Daha sonra başarımı birçok veri kümesi üzerinde kanıtlanmış AlexNET [16] mimarisinin parametreleri transfer öğrenme yaklaşımıyla yeni bir ESA mimarisine aktarılmıştır. Sonraki aşamada, veri kümesindeki örnek sayısı ÇÜA yöntemiyle genişletilmiştir. Son aşamada ESA mimarisi üç farklı veri kümesiyle (HAM10000, HAM10000+klasik veri artırımı, HAM10000+ÇÜA) ayrı ayrı eğitilmiştir. Elde edilen sonuçlar doğruluk ve zaman açısından değerlendirilmiştir. Ayrıca HAM10000 veri seti için literatürde önerilen derin öğrenme modeli ile önerdiğimiz ÇÜA+ESA karşılaştırılarak detaylı sonuçlar tartışılmaktadır.

\section{Cilt Lezyonlarının Sınıflandırılması}

$\mathrm{Bu}$ bölümde kullanılan veri kümesi tanıtılmaktadır. Verinin sınıfları ve anlamları ve ayrıca verinin sınıfsal dağılımı detaylıca açıklanmaktadır. Daha sonra AlexNET mimarisinden bahsedilmektedir. Son olarak da AlexNET den veri transfer yaklaşımları detaylandırılmaktadır.

\subsection{Cilt Lezyonlarının Sinıflandırılması}

HAM10000 [15] veri kümesinde bulunan cilt lezyon imgeleri, farklı coğrafik bölgelerde yaşayan ve veri kümesindeki cilt hastalıklarına sahip insanlardan elde edilmiştir. Veri kümesi yedi farklı lezyon türüne (Akiec, Bcc, Bkl, Df, Nv, Mel ve Vasc) ait imgeler içermektedir. Her bir lezyon türünün anlamı ve veri kümesindeki adedi Tablo 1'de ifade edilmektedir. Veri kümesi toplamda 10015 adet 650x400x3 boyutunda RGB lezyon imgesini içermektedir. Bu veri kümesini diğer cilt kanseri veri kümelerinden ayıran en önemli özellik, her bir kategoride yer alan lezyon adedinin yüksek miktarda olmasıdır. Dahası bu veri kümesindeki imgelerde, lezyonlu bölge üzerinde bulunabilen kıl ve güneş yanığı gibi lezyon görselini bozan dış etkenler tanı ve sınıflandırmayı olumsuz yönde etkilediği not edilmiştir. 
Tablo 1. HAM10000 cilt lezyonu imgeleri veri kümesi

\begin{tabular}{|c|c|c|c|}
\hline NO & TÜR & AÇIKLAMA & ADET \\
\hline 1 & Akiec & Ameliyat olmaya gerek kalmadan lokal tedavi yapılabilir & 327 \\
\hline 2 & Bcc & $\begin{array}{l}\text { Bazal hücreli karsinom nadiren metastaz yapan ancak tedavi edilmediğinde yıkıcı olarak büyüyen } \\
\text { epitel cilt kanserinin yaygın bir çeşidi }\end{array}$ & 514 \\
\hline 3 & Bkl & İyi huylu keratoz olarak bilinir. Görsel olarak ayırt edilmesi güçtür & 1099 \\
\hline 4 & Df & Dermafibroma olarak bilinen iyi huylu bir cilt lezyonu & 115 \\
\hline 5 & Nv & $\begin{array}{l}\text { İyi huylu neoplazmalardır ve melanomun aksine renk ve yapı dağılımı bakımından genel olarak } \\
\text { simetrik }\end{array}$ & 6705 \\
\hline 6 & Mel & Melenom ile kötü huylu bir neoplazma & 1113 \\
\hline 7 & Vasc & $\begin{array}{l}\text { Vasküler cilt lezyonlarıdır. Kırmızı ve mor renkli lekeler olarak bilinirler. İyi huylu yapılar olarak not } \\
\text { edilmiş }\end{array}$ & 142 \\
\hline & & $\begin{array}{lc}\text { Toplam } \\
\end{array}$ & 10015 \\
\hline
\end{tabular}

\subsection{AlexNET}

Sınıflandırma başta olmak üzere birçok bilgisayarlı görü alanında kullanılan ESA, klasik sinir ağlarından farklı olarak, özellik çıkarma ve sınıflandırma katmanlarını içermektedir. Evrişim katmanı konvolüsyon (convolution), aktivasyon fonksiyonu (ReLU) ve havuzlama (pooling) katmanlarını içermekte ve yüksek boyutlu veriden düşük boyutlu özniteliklerin çıkarılmasını sağlamaktadır. Probleme özgü olarak evrişim katmanının ardışıl bağlanma sayısı modelin derinliğini değiştirmektedir. Sınıflandırma katmanı ise düşük boyutlu öznitelikler ile kategorilerin eşleşmesini sağlar ve genellikle tam bağlı bir YSA mimarisine sahiptir. Krizhevsky ve diğerlerinin önerdiği ve AlexNET olarak bilinen mimari, 5 evrişim katmanına ve 3 tam bağlı sınırlandırıcıya (60 milyon ağırlıklı ve 650000 hücreli) sahiptir. Şekil 1'de mimari detaylı olarak gösterilmektedir. Bu mimari ImageNET veri seti ile eğitildikten sonra öğrenilen bilgiler (ağıllıklar) farklı problem çözümlerinde transfer öğrenme teknikleri yardımıyla kullanılmış ve yüksek başarım elde edildiği gösterilmiştir [16], [17].

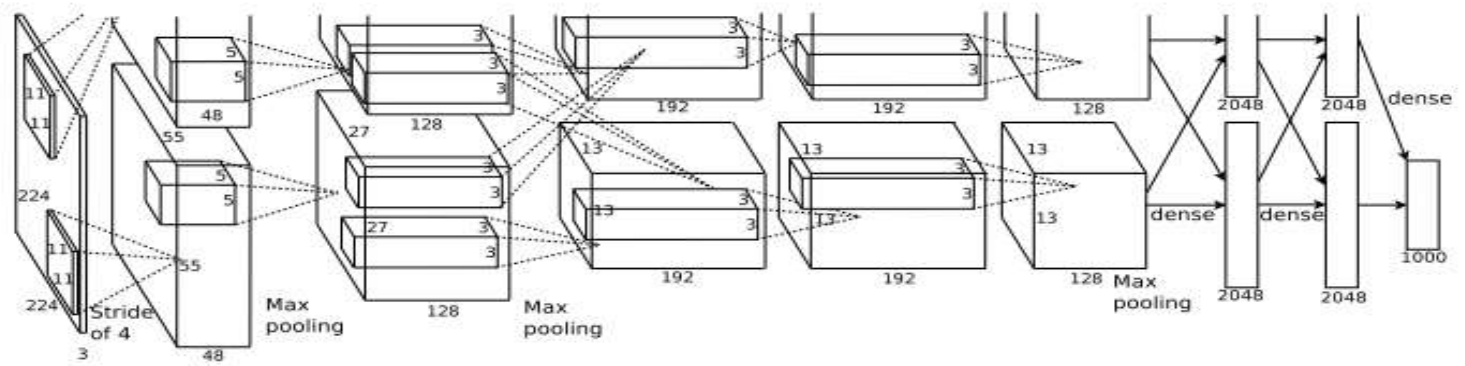

Şekil 1. AlexNET mimarisi [16].

Şekil 1'de gösterilen mimarinin giriş verisi $227 \times 227$ boyutunda 3 kanallı bir imgedir. Daha sonra beş katmanı özellik çıkarıcı evrişim katmanı bulunur. Son olarak bu evrişim katmanlarının bağlandığı modelin kullanım amacına göre sınıflama gibi işlemleri yürüten tam bağlı katmandan oluşmuştur.

AlexNET, ImageNET veri kümesini sınıflandırabilmek için önerilmiş bir mimaridir. ImageNET [18] (1.000 sınıf ve 1.000 .000 imge) kedi, köpek, kuş ve bunun gibi veri sınıflarının bulunduğu veri kümesidir. AlexNET ağırlıklarının farklı problemlerde kullanabilmek için transfer öğrenme yaklaşımlarından faydalanmak gerekmektedir. Kısaca transfer öğrenme yapay öğrenme sistemlerinin eğitim aşamasıyla öğrendiği bilgiyi farklı veya benzer problem çözümlerinde kullanılmasını inceleyen öğrenme yaklaşımıdır. ESA için kullanılan 2 farklı transfer öğrene yaklaşımı bulunmaktadır. Bunların ilki özellik çıkarıcı yöntemdir. Bu yaklaşım elimizdeki problem için tasarlanmış bir ESA'nın evrişim katman ağırlık değerlerinin eğitilmiş bir ESA'dan alınması ve eğitim işleminin sadece sınıflandırma katmanında yapılması şeklindeki kullanımdır. Sınıflandırma katmanı yapay sinir ağı olabildiği gibi farklı bir yöntem (örn. DVM) olabilir. Böylece eğitilmiş ESA'nın özellik çıarma kabiliyeti yeni ESA'ya aktarılmaktadır. İkinci yaklaşım ise kısmi özellik çıkarıcı yöntemdir. Eğitilmiş ESA'nın evrişim katmanındaki ağırlıkların bütünü yerine belirli bir kısmının yeni ESA'ya aktarılması ve yeni ESA'daki kalan evrişim katmanıyla birlikte sınıflandırıcı katmanın yeni eğitim kümesi kullanılarak güncellenmesi şeklindeki kullanımdır. Bu iki transfer öğrenme yönteminin AlexNET üzerindeki performansı etkileyen en temel 
kriterler, AlexNET'e uygulanacak yeni veri kümesinin veri sayısı ve yeni veri kümesinin ImageNET'e olan benzerliğidir [19].

AlexNET'den transfer edinilen ağ parametreleri iki farklı şekilde kullanılabilmektedir: 1) özellik çıkarıcı yaklaşım: Yeni veri kümesindeki sınıf sayısına göre AlexNET mimarisindeki tam bağlı YSA katmanının güncellenmesi. Eğitim işlemi sadece yeni eklenen tam bağlı YSA üzerinde gerçekleştirilir (SGD, MiniBatch: 256, $\alpha$ : 0,001). Bu YSA, her bir eğitim döngüsünde 256 adet gruplara ayrılmış özellik vektörünün 0,001 değerli öğrenme sabitine göre geriye yayılımlı olarak eğitilmesiyle sonuçlandırılır. 2) Kısmi özellik çıkarıcı yaklaşımda ise ilk 7 katmanın çıkışı vektörleştirilir (1x4096 boyut) ve DVM ile sınıflandırılır. DVM parametreleri veri kümesine göre değişkenlik göstermektedir. HAM10000 veri kümesi için çok sınıflı liner DVM kullanılmıştır.

\section{Gerçek Cilt Lezyon İmgelerinden Veri Üretilmesi}

Yapay öğrenmede eğitim işlemi yapan modellerin temel problemi her zaman yeterli sayıda etiketlenmiș veri bulunmamasıdır. Cilt lezyon veri kümesi HAM10000 için 7 farklı kategoride veri sayıları değişkenlik göstermektedir. $\mathrm{Bu}$ değişkenlik sınıflama başarısını olumsuz yönde etkilemektedir. Tanı ve sınıflama performansını artırmak için 2 farklı yaklaşım belirlenmektedir. İlki klasik veri artırım yöntemi ve diğeri veri kümesi için önerilen ÇÜA yapısıdır.

\subsection{Klasik Üretim}

Eğitim kümesindeki imgeler üzerinde aşağıda listelenen geometrik/renk dönüşümleri bağımsız bir şekilde gerçekleştirilmekte ve eğitim kümesinde olmayan yeni imgeler üretilerek veri artırımı yapılmaktadır.

- Çevirme (Flipping): Yatay yönde çevirme

- Döndürme (Rotation): Rastgele bir merkezden döndürme

- Kırpma (Cropping): Belirlenen bir bölgenin kırpılması ve yeniden boyutlandırılması

- Kaydırma (Shifting): Sağa sola kaydırılması

- Renk Seğirme (Color jittering): Rasgele parlaklık, doygunluk ve zıtlığın değiştirilmesi

- Gürültü Ekleme (Noise adding): Gürültünün eklenmesi

Klasik imge üretim işleminin en önemli faydalarından biri, hiç şüphesiz sistemin aşırı öğrenmesini ve ezberlemesini engellemesidir.

\subsection{Derin Evrişimsel Sinir Ă̆ı ile Üretim}

ÇÜA, gerçek imgeler ile ayırt edilemeyecek sentetik imge üreten ilk başarılı yapay öğrenme tabanlı modellerdir. ÇÜA'lar sanat, moda, tıp gibi çeşitli alanlarda uygulanmaktadır [20]. 2014 yılında Goodfellow [3] tarafından geliştirilen bu teknik, üretici (generator, G), ayırıcı (discriminator, D) olmak üzere iki farklı ağdan oluşur. Sistem mimarisi Şekil 2' gösterilmiştir. G, rastgele aldığı bir gürültü ile çeşitli işlemlerden sonra yeni imgeler üreterek bu imgelerr gerçekmiş gibi D’yi hatalı sınıflama yapmasına çalışırken, D ise giriş olarak aldığ 1 gerçek ve yeni üretilmiş imgeleri sınıflandırmaya (gerçek(1)/yeniüretilmiş(0)) çalışır.

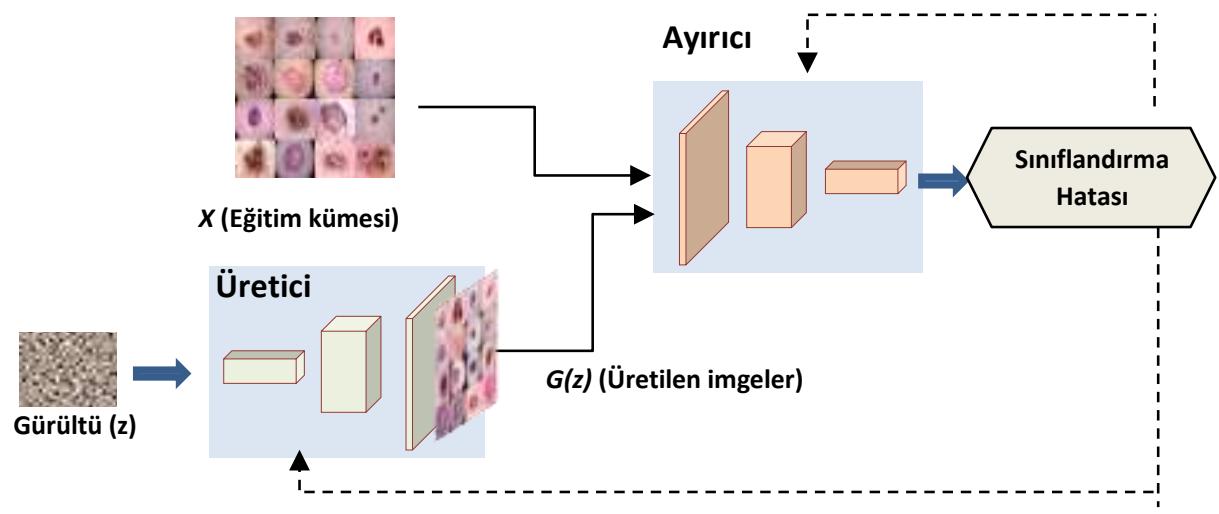

Şekil 2. Klasik ÇÜA mimarisi 
Tablo 2. DEÇÜA’’n G ve D ağlarındaki katmanları

\begin{tabular}{|c|c|c|c|c|c|c|}
\hline Ăg & Katman & $\begin{array}{c}\text { Özellik } \\
\text { Haritası } \\
\end{array}$ & Çıkış Boyutu & $\begin{array}{c}\text { Filtre } \\
\text { Boyutu }\end{array}$ & Adım & Aktivasyon \\
\hline \multirow{13}{*}{ 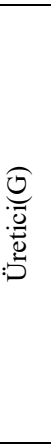 } & Tam Bağlı Katman(Dense) & - & 32768 & - & - & ReLU \\
\hline & Yeniden Boyutlandırma(Reshape) & - & $16 \times 16 \times 128$ & - & - & - \\
\hline & 2B Evrișim Katmanı (Conv2D) & 128 & $16 \times 16 \times 128$ & $4 \times 4$ & 1 & ReLU \\
\hline & Normalizasyon(BatchNormalizasyon) & - & $16 \times 16 \times 128$ & - & - & ReLU \\
\hline & Büyütme(UpSampling2D) & - & $32 \times 32 \times 128$ & $2 \times 2$ & - & - \\
\hline & 2B Evrişim Katmanı (Conv2D) & 128 & $32 \times 32 \times 128$ & $5 \times 5$ & 1 & ReLU \\
\hline & Normalizasyon(BatchNormalizasyon) & - & $32 \times 32 \times 128$ & - & - & ReLU \\
\hline & Büyütme(UpSampling2D) & - & $64 \times 64 \times 128$ & $2 \times 2$ & - & - \\
\hline & 2B Evrişim Katmanı (Conv2D) & 128 & $64 \times 64 \times 128$ & $5 \times 5$ & 1 & ReLU \\
\hline & Normalizasyon(BatchNormalizasyon) & - & $64 \times 64 \times 128$ & - & - & ReLU \\
\hline & 2B Evrişim Katman1 (Conv2D) & 128 & $64 \times 64 \times 128$ & $5 \times 5$ & 1 & ReLU \\
\hline & Normalizasyon(BatchNormalizasyon) & - & $64 \times 64 \times 128$ & - & - & ReLU \\
\hline & 2B Evrişim Katmanı (Conv2D) & 3 & $64 \times 64 \times 3$ & $5 \times 5$ & 1 & ReLU \\
\hline \multirow{12}{*}{ 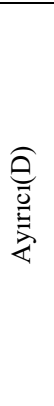 } & Giriş(İnput) & - & $64 \times 64 \times 3$ & - & - & - \\
\hline & 2B Evrişim Katmanı (Conv2D) & 128 & $64 \times 64 \times 128$ & $3 \times 3$ & 1 & LeakyReLU(alpha $=0.1$ ) \\
\hline & Normalizasyon(BatchNormalizasyon) & - & $64 \times 64 \times 128$ & - & - & LeakyReLU(alpha $=0.1)$ \\
\hline & 2B Evrişim Katmanı (Conv2D) & 128 & $32 \times 32 \times 128$ & $4 \times 4$ & 2 & LeakyReLU(alpha $=0.1)$ \\
\hline & Normalizasyon(BatchNormalizasyon) & - & $32 \times 32 \times 128$ & - & - & LeakyReLU(alpha $=0.1)$ \\
\hline & 2B Evrişim Katmanı (Conv2D) & 128 & $16 \times 16 \times 128$ & $4 \times 4$ & 2 & LeakyReLU(alpha $=0.1)$ \\
\hline & Normalizasyon(BatchNormalizasyon) & - & $16 \times 16 \times 128$ & - & - & LeakyReLU(alpha $=0.1$ ) \\
\hline & 2B Evrişim Katmanı (Conv2D) & 128 & $8 \times 8 \times 128$ & $4 \times 4$ & 2 & LeakyReLU(alpha $=0.1$ ) \\
\hline & Normalizasyon(BatchNormalizasyon) & - & $8 \times 8 \times 128$ & - & - & LeakyReLU(alpha=0.1) \\
\hline & Vektöre Dönüştürme(Flatten) & - & 8192 & - & - & \\
\hline & Tam Bağlı Katman(Dense) & - & 512 & - & - & ReLU \\
\hline & Tam Bağlı Katman(Dense) & - & 1 & - & - & Sigmoid \\
\hline
\end{tabular}

Üretici eğitimi [20][21]:

1. G, giriş olarak rastgele gürültü (z) vektörünü alır ve G(z) yeni imgesini üretir.

2. G'nin eğitimi denklem 1'e göre yapıılır [3]. G ağı D'nin hatasını maksimum düzeye çıkarmak X'e benzer imgeler üretir. Denklem 1'deki m değeri giriş imge sayısıdır.

$\nabla_{\theta_{g}}=\frac{1}{m} \sum_{i=1}^{m} \log \left(1-D\left(G\left(z^{i}\right)\right)\right)$

Ayırıcı eğitimi [20][21]:

1. Giriş olarak $X$ gerçek imgeyi ve $G(z)$ yeni üretilmiş imgeyi alır. Denklem 2'deki m değeri giriş imge sayısidır

2. D'nin eğitimi denklem 2'ye göre yapılır [3].

$\nabla_{\theta_{d}}=\frac{1}{m} \sum_{i=1}^{m}\left[\log D\left(x^{i}\right)+\log \left(1-D\left(G\left(z^{i}\right)\right)\right)\right]$

Radford ve arkadaşları [22] tarafından geliştirilen, DEÇÜA, üretici ve ayırıcı mimarilerine bazı kısıtlamalar eklemiş ve daha kaliteli imgelerin üretilmesini sağlamıştır. Bu kısıtlamalar:

- Havuzlama katmanı yerine D içerisinde sıçramalı konvolüsyon (strided convolutions), G içinde kademeli sıçramalı konvolüsyon (fractional-strided convolutions) işlemini kullanır.

- Hem D hem de G için normalleştirme (BatchNorm) kullanır.

- Daha derin mimariler için tam bağlı gizli katman kullanmaz.

- G ağının katmanlarında ReLU aktivasyon fonksiyonu kullanılır (çıkış katmanı tanh).

- D'nin tüm katmalarında aktivasyon fonksiyonu olarak LeakyReLU kullanılır.

DEÇÜA yönteminin $G$ ve $D$ ağlarındaki katmanlar Tablo 2'de verilmektedir. $G$ ağı, 1 tam bağlı YSA (128xİmgeBoyutu) ve 7 evrişim katmanı içermektedir. D ağı, 4 evrişim, 1 düzleştirme ve 2 tam bağlı YSA 
katmanı içermektedir. D'deki ilk tam bağlı YSA katmanında 512 hücre, ikincisinde 1 hücre bulunmaktadır. DEÇÜA mimarisi her sınıf için 6.000 iterasyon eğitilmiştir. Eğitim süresi eğitim veri setine bağlı olarak ortalama 8-10 saat sürmüştür. Optimizasyon algoritması olarak Adam $(\alpha=0.0002, \beta=0.5)$ [23] kullanılmıştır. Adagrad ve RMSprop optimizasyon algoritmalarının avantajlarını göz önünde bulundurularak geliştirilen bu algoritma aynı zamanda momentum değişikliklerini de hesaplar. $\alpha$ öğrenme katsayısını, $\beta$ ise moment hesaplamaları için bozulma oranını ifade etmektedir. Uygulamasının basit, hesaplama maliyetinin ve bellek/parametre gereksiniminin düşük olması, DEÇÜA yöntemini büyük veri kümeleri ve gürültülü olan problemler için de uygundur [23].

\section{Deneysel Sonuçlar}

Deneysel uygulamalar Matlab (2018b) ortamında ve Pyton programlama dili kullanılarak geliştirilmiştir. Pyton programlama dili ile Keras Kütüphanesi kullanılarak ÇÜA mimarisi tasarlanmıştır. Ayrıca Matlab 2018b uygulaması ile AlexNET mimarisinin ağırlıkları transfer öğrenme yoluyla alınarak cilt lezyonlarının sınıflama işlemi gerçekleştirilmiştir. Uygulamaların koştuğu sunucu, 20 çekirdekli 2 işlemcili, Nvidia Quard 4000 GPU hızlandırıcı kartı bileşenlerine sahiptir.

Birinci deneysel çalışmada, HAM10000 veri kümesindeki imgeler kullanılarak klasik üretim tekniğiyle yeni imgeler üretilmiştir. İmgeler dikey eksende \pm 30 piksel aralığında çevrilip, 0-360 derecede rasgele döndürülmekte ve \%10 oranında ölçeklendirme yapılmaktadır. Şekli 3’te üretilen yeni imgeler gösterilmektedir.

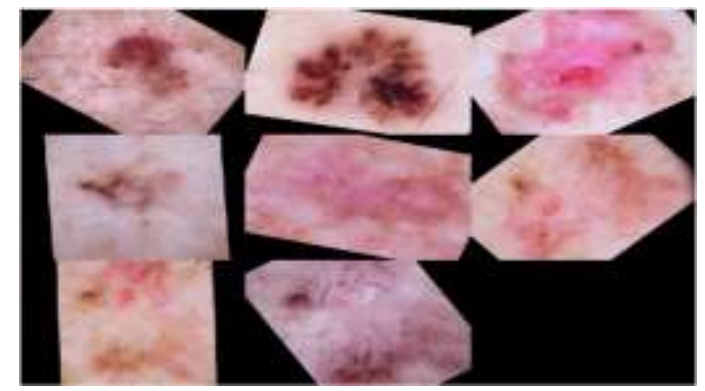

Şekil 3. Klasik üretim tekniği ile üretilen imgeler

İkinci deneysel çalışmada, DEÇÜA algoritması kullanılarak HAM10000 veri kümesine yeni imgeler eklenmiştir. DEÇÜA mimarisinin eğitimi tamamlandıktan sonra her sınıftan 8.000 adet toplam imge olacak şekilde üretim yapılmıştır. Üretilen örnek imgeler Şekil 4'te gösterilmektedir.
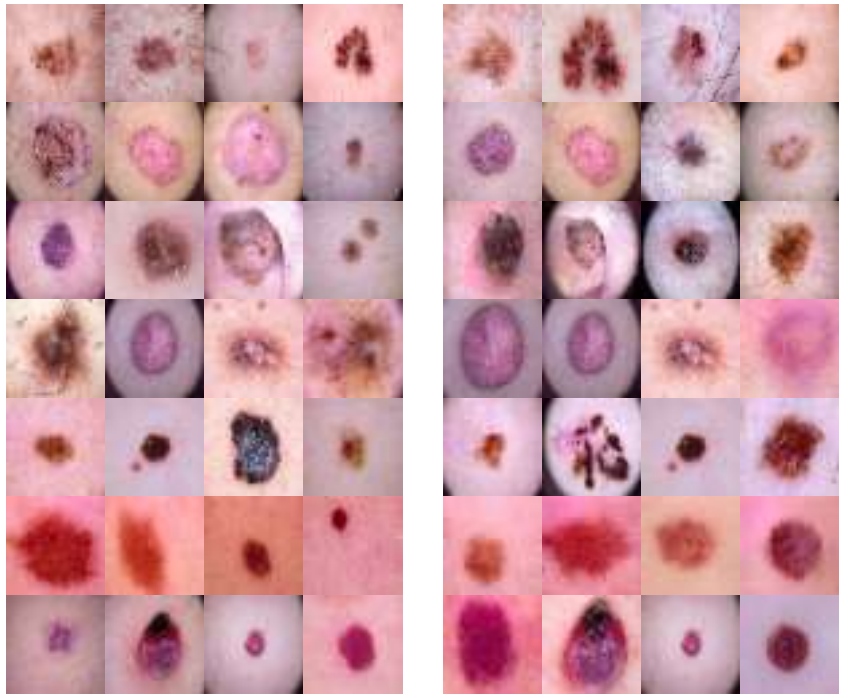

Şekil 4. DEÇÜA ile üretilen yeni imgeler (sol) gerçek imgeler (sağ) üretilen yeni imgeler 
Üçüncü deneysel çalışmanın amacı aşağıda ifade edilen etkileri ortaya çıkarmaktır:

1. İki farklı parametre transferi yaklaşımının (AlexNET’ten) ESA doğruluğuna ve sınıflandırma maliyetine etkisi

2. İki farklı imge üretim tekniğinin ESA doğruluğuna ve sınıflandırma maliyetine etkisi

İki farklı parametre transferi yaklaşımı (kısmi ve tam) sonucunda ortaya iki farklı sınıflandırıcı (ESA1 ve ESA2) çıkarmaktadır. Her iki sınıflandırıcı üç farklı veri kümesiyle (HAM10000, HAM10000+ Klasik Üretim ve HAM10000+ÇÜA) eğitilerek doğruluk ve harcanan zaman sonuçları gözlemlenmiștir. Her iki ESA'nın doğruluk değeri Denklem 3 'te gösterildiği gibi hesaplanmaktadır:

$$
\text { Doğruluk }=\frac{\sum T P}{\text { Toplam Lezyon Sayısl }}
$$

TP değişkeni, sınıflardaki doğru tahmin edilen lezyon sayısını ifade etmektedir. Böylece doğruluk değeri, her bir sınıfta tahmin edilen doğru veri sayısının toplam lezyon sayısına oranıdır.

Tablo 3. Transfer öğrenme ve imge üretim yaklaşımlarının etkisi

\begin{tabular}{|c|c|c|c|c|}
\hline \multirow{2}{*}{ Veri kümesi } & \multicolumn{4}{|c|}{ ESA1 (kısmi parametre transferi) } \\
\cline { 2 - 5 } & Ĕ̈itim veri sayısı & Test veri sayısı & Doğruluk (\%) & Zaman (dk) \\
\hline HAM10000 & 7.000 & 3.000 & 76,55 & 13,58 \\
\hline HAM10000 + Klasik üretim & 56.000 & 3.000 & 80,25 & 25,05 \\
\hline HAM10000 + ÇÜA & 56.000 & 3.000 & $\mathbf{9 3 , 1 4}$ & 100 \\
\hline & \multicolumn{4}{|c|}{ ESA2 (tam parametre transferi) } \\
\cline { 2 - 5 } & Ĕ̈itim veri sayısı & Test veri sayısı & Doğruluk (\%) & Zaman (dk) \\
\hline HAM10000 & 7.000 & 3.000 & 73,5 & 3,55 \\
\hline HAM10000 + Klasik üretim & 56.000 & 3.000 & 74,32 & 17,6 \\
\hline HAM10000 + ÇÜA & 56.000 & 3.000 & $\mathbf{9 0}$ & 73.4 \\
\hline
\end{tabular}

Tablo 3'te elde edilen sonuçlar incelendiğinde, imge üretim yöntemleri sonucu eğitim veri sayısı 7.000'den 56.000'e yükselmiştir. ESA1 ve ESA2 sınıflandırıcıların her ikisi de en yüksek doğruluğa HAM10000+ÇÜA veri kümesiyle ulaşabilmektedir. Ancak Ham10000+ÇÜA veri kümesinin eğitimi için harcanan süre diğer iki veri kümesinden fazla çıkmıştır. Çünkü Gan veri üretim adımının yürütme zamanı ESA ların sınıflama zamanlarına eklenerek sunulmuştur. Bununla birlikte ESA1'in doğruluk değeri ESA2'den daha yüksek çıkmıştır. Böylece kısmi parametre transferinin yeterli veri sayısı olduğunda daha makul olduğu görülmüştür.

Literatürde HAM10000 veri kümesindeki hastalıkların teşhisi için makine öğrenmesi temelli çeşitli çözüm önerileri sunulmuştur. Bunlardan HAM10000 veri kümesi için ÇÜA kullanarak ESA sınıflama performansını artırmaya çalışan yöntem [6] ile Res50 ve Res100 ağlarını kullanarak özellik çıkarımı yapan ve veri kümesini sınıflayan [14] nolu çalışmanın toplam doğruluk performansı Tablo 4'te önerilen yöntem ile kıyaslanmıştır.

Tablo 4. Önerilen yöntem ile [14] nolu referansın toplam doğruluk üzerine karşılaştırma sonucu

\begin{tabular}{|l|c|}
\hline \multicolumn{1}{|c|}{ Yöntem } & Doğruluk (\%) \\
\hline ESA1 (kısmi parametre transferi) & 90 \\
\hline ESA2 (tam parametre transferi) & 93,14 \\
\hline ESA3 ([14]'deki yöntem) & 89,8 \\
\hline ESA4([6'daki yöntem] & 71,7 \\
\hline
\end{tabular}

Tablo4'te DEÇÜA ile veri üretimi yapılmış AlexNET ağırlıklarıyla kısmı parametre transferi ile tam parametre transferi yapan önerilen yöntem literatürdeki [14] nolu yönteme göre toplam doğruluk üzerinde \%1\%3 iyileşme sağladığı görülmüştür. Ayrıca ÇÜA kullanarak veri artırımı yapan [6] nolu referanstaki yönteme göre önerilen yöntemin yaklaşık \%30 toplam doğrulukta başarım sağladığı görülmüsşür.

\section{Sonuçlar}

$\mathrm{Bu}$ çalışmada klasik veri arıtımı ve ÇÜA tabanlı veri üretim yönteminin transfer öğrenmenin sınıflama başarımına etkisi HAM10000 veri kümesine uygulanarak detaylandırılmıştır. Önerilen DEÇÜA tabanlı veri üretim yönteminin iki farklı transfer öğrenme yaklaşımında, klasik veri arıtırım yöntemine göre $\% 15$ ila \%20 
arasında sınıflama doğruluğunu arttırdığı gözlemlenmiştir. Çalışmada, HAM10000 veri kümesi, DEÇÜA tabanlı veri artırımından sonra kısmi parametre transferi ile en yüksek cilt lezyon sınıflama doğruluğu elde edilmiştir. Cilt lezyonları için önerilen bu yöntem ile HAM1000 veri kümesi için yaklaşık \%93 lük sınıflama doğruluk başarımına ulaşılmıştır. HAM10000 veri kümesi için literatürde sunulan yöntemlerle kıyaslanarak önerilen yöntemin toplam doğruluk üzerinde önemli ölçüde üstünlük gösterdiği detaylandırılmıştır. Makalede özellikle bilgisayar bilimcilerin, tıp alanındaki veri sayısı kısttlı problemlere ÇÜA ile veri üretimi yaparak çözüm üretebilecekleri gösterilmiştir. Gelecekteki çalışmamızda, cilt lezyonlarını giriş verisi alarak hastalık tespitini yapabilen karar destek sisteminin geliştirilmesi planlanmıştır.

\section{Kaynaklar}

[1] Frid-Adar M, Diamant I, Klang E, Amitai M, Goldberger J, and Greenspan H. GAN-based synthetic medical image augmentation for increased CNN performance in liver lesion classification. Neurocomputing 2018; 321: 321-331.

[2] Shijie J, Ping W, Peiyi J, and Siping H. Research on data augmentation for image classification based on convolution neural networks. Chinese Autom. Congr. CAC; 2016; China. 4165-4170.

[3] Goodfellow I. J, Pouget-Abadie J, Mirza M, Xu B, Warde-Farley D, Ozair S, Courville A, Bengi Y. Generative Adversarial Networks. arXiv:1406.2661v1 2014; 1-9.

[4] Isola P, Zhu J. Y, Zhou T, and Efros A. A. Image-to-image translation with conditional adversarial networks. Proc. 30th IEEE Conf. Comput. Vis. Pattern Recognition CVPR 2017; USA: 5967-5976.

[5] Yeh RA, Chen C, Yian Lim T, Schwing A. G, Hasegawa-Johnson M, and Do M. N. Semantic image inpainting with deep generative models. Proc. - 30th IEEE Conf. Comput. Vis. Pattern Recognition CVPR; 2017; USA: 6882-6890.

[6] Bisla D, Choromanska A, Stein J. A, Polsky D, and Berman R. Skin Lesion Segmentation and Classification with Deep Learning System. arXiv:1902.06061v1 2019; 1-6.

[7] Han C et al. GAN-based synthetic brain MR image generation. Proc. - Int. Symp. Biomed. Imaging; 2018; USA. 734 738.

[8] Bi L, Feng D, and Kim J. Improving Automatic Skin Lesion Segmentation using Adversarial Learning based Data Augmentation. arXiv:1807.08392 2018; 1-6.

[9] Xue Y, Xu T, Zhang H, Long L. R, and Huang X. SegAN: Adversarial Network with Multi-scale L 1 Loss for Medical Image Segmentation. Neuroinformatics 2018;16: 383-392.

[10] Siegel RL, Miller KD, and Jemal A. Cancer statistics, CA. Cancer J. Clin 2019; 69: 7-34.

[11] Vestergaard ME, Macaskill P, Holt PE, and Menzies SW. Dermoscopy compared with naked eye examination for the diagnosis of primary melanoma: A meta-analysis of studies performed in a clinical setting. Br. J. Dermatol 2008; 159: 669-676.

[12] Codella NCF et al. Skin lesion analysis toward melanoma detection: A challenge at the 2017 International symposium on biomedical imaging (ISBI), hosted by the international skin imaging collaboration (ISIC). Proc. - Int. Symp. Biomed. Imaging 2018; USA: 168-172.

[13] Brinker TJ et al. Deep learning outperformed 136 of 157 dermatologists in a head-to-head dermoscopic melanoma image classification task. Eur. J. Cancer; 2019; 113: 47-54.

[14] Khan MA, Saba T, and Sharif M. Multi-Model Deep Neural Network based Features Extraction and Optimal Selection Approach for Skin Lesion Classification. Int. Conf. Comput. Inf. Sci; 2019; Saudi Arabia: 1-7.

[15] Tschandl P, Rosendahl C, and Kittler H. Data descriptor: The HAM10000 dataset, a large collection of multi-source dermatoscopic images of common pigmented skin lesions Sci. Data 2018; 5:1-9.

[16] Krizhevsky A. and Hinton G. E. ImageNet Classification with Deep Convolutional Neural Networks. Adv. Neural Inf. Process. Syst. 2012;1907(1105): 1-9.

[17] Akçay M. E, Kundegorski Samet, Devereux M, and Breckon T. P. Transfer Learning Using Convolutional Neural Networks for Object Classification Within X-Ray Baggage Security Imagery. International Conference on Image Processing (ICIP) 2016; USA: 23: 1057 - 1061.

[18] “Image.net.” Erişim Tarihi: 15/05/2019.

[19] Yosinski J, Clune J, Bengio Y, and Lipson H. How transferable are features in deep neural networks? Adv. Neural Inf. Process. Syst. 2014;2: 3320-3328.

[20] Lange J, Bok V. GANs in Action, MEAP. Shelter Island, NY / USA: Manning Publications, 2018.

[21] Goodfellow I. Tutorial: Generative Adversarial Networks. arXiv:1701.00160v4 2016;1-57.

[22] Radford A, Metz L, and Chintala S. Unsupervised Representation Learning with Deep Convolutional Generative Adversarial Networks International Conference on Image Processing (ICIP) USA; 2015: 1-16.

[23] Kingma DP and Adam JBa.: A Method for Stochastic Optimization 2014; 1-15. 